\title{
A CURVATURE OBSTRUCTION TO COVERING DEFORMATIONS OF CONNECTIONS BY METRIC DEFORMATIONS
}

\author{
PAUL E. EHR LICH
}

ABSTRACT. A curvature obstruction to covering a deformation of connections of a Levi-Civita connection of a Riemannian metric by a metric deformation is derived and used to study deformations of connections related to the deformation of connections arising from a conformal deformation of a Riemannian metric.

In this note we assume familiarity with the difference tensor of two connections, as explained for instance in [5, \$5.4]. Everything will, for convenience, be taken to be $C^{\infty}$. Given a manifold $M^{n}, n \geq 2$, and a Riemannian metric $g$ for $M$, a conformal deformation $g(t)=e^{2 t f} g$ of $g$ results in the following classical formula for the Levi-Civita connection $D^{t}$ of $g(t)$ :

$$
D_{X}^{t} Y=D_{X} Y+t(\xi(X) Y+\xi(Y) X-g(X, Y) \operatorname{grad} f)
$$

where $\xi:=d f$ and $D$ is the Levi-Civita connection associated to $g$. Conformal deformations have proved useful in deforming Ricci curvature [1], [4], and in considering the problem of conformal transformations [3], [6]. However, in [4] we saw that at least at first order, conformal deformations are not good for perturbing sectional curvature.

This fact together with the form of the difference tensor $D^{t}-D$ in (1) suggests that we consider whether the deformations of the Levi-Civita connection $D$ of $g$ given by

$$
D_{X}^{t} Y=D_{X} Y+t(\xi(X) Y+\xi(Y) X)
$$

and

$$
D_{X}^{t} Y=D_{X} Y+\operatorname{tg}(X, Y) Z
$$

where $Z$ is a fixed vector field on $M$, can be covered by metric deformations $g(t)$ of $g$. Note that (2) and (3) are perhaps the simplest natural deformations of connections involving vector fields. In particular, deformation (2) is just

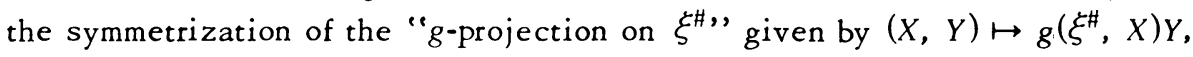
where we use the notation $\xi^{\#}$ for the vector field associated to the 1 -form $\xi$ by $g$. (Explicitly, $g\left(\xi^{\#}, X\right):=\xi(X)$.)

It is curious that (2), which is just the first two terms of the conformal

Received by the editors July 17, 1974 .

AMS (MOS) subject classifications (1970). Primary 53C20. 
deformation (1), is precisely the tensor discovered by Weyl in studying projectively equivalent connections, and that at least in the case of constant curvature, the deformations (2) and (3), which do not appear to be much alike, fail to be covered even at first order by metric deformations for the same reasons (Proposition $1 \leftrightarrow$ Proposition 4, and Proposition $3 \leftrightarrow$ Proposition 5).

In $\$ 1$ we derive the "curvature obstruction" for a deformation of a LeviCivita connection through torsion free connections to be covered by a metric deformation. In $\$ 2$ we use this curvature obstruction to study the deformations (2) and (3) on constant curvature manifolds.

We use the sign convention $R(X, Y)=\left[D_{X}, D_{y}\right]-D_{[X, Y]}$ for the curvature tensor. We use the form of the Lie derivative operator $\delta^{*}: A^{1}(M) \rightarrow S^{2}(M)$ defined in [2, $\{2]$. We will also use the notation of [2] for various bundles and differential operators arising in connection with metric deformations. In the calculations and formulas below, we will usually write $\langle$,$\rangle for a fixed Rie-$ mannian metric $g$.

1. The curvature obstruction. Let $D$ be a fixed connection on $M$. Let $t \mapsto D^{t}$ be a deformation of connections through $D$ (thus $D^{0}=D$ ), analytic in $t$ of at least order 2. We may write

$$
D^{t}=D+C^{t}=D+t C^{1}+\left(t^{2} / 2\right) C^{2}+\cdots
$$

where the $C^{i}$ are tensors. We call $C^{\prime}:=\left.(d / d t) D^{t}\right|_{t=0}$ the first order difference tensor of the deformation $t \mapsto D^{t}$. (Of course $C^{\prime}=C^{1}$.)

Let $R^{t}$ be the curvature tensor of $D^{t}$. A calculation shows

$$
R^{t}(x, y) z=R(x, y) z+D_{x}\left(C^{t}\right)(y, z)-D_{y}\left(C^{t}\right)(x, z)-\left[C^{t}(x,-), C^{t}(y,-)\right](z)
$$

where

$$
\left[C^{t}(x,-), C^{t}(y,-)\right](z)=C^{t}\left(x, C^{t}(y, z)\right)-C^{t}\left(y, C^{t}(x, z)\right) .
$$

If we define $r\left(D^{t}\right)=r\left(C^{\prime}\right):=\left.(d / d t) R^{t}\right|_{t=0}$, then as was pointed out to us by J. Simons,

$$
r\left(D^{t}\right)(x, y, z)=r\left(C^{\prime}\right)(x, y, z)=D_{x}\left(C^{\prime}\right)(y, z)-D_{y}\left(C^{\prime}\right)(x, z) .
$$

Thus considering deformations of curvature tensors at first order from the viewpoint of deformations of connections leads to the relatively simple differential system of Simons' type

$$
(\sigma h)(x, y, z):=\left(D_{x} h\right)(y, z)-\left(D_{y} h\right)(x, z)
$$

( see $[2, \$ 6.2])$. This simplicity contrasts with the formula for $R^{\prime}$ when $t$ $\mapsto D^{t}$ is a deformation of $D$ through Levi-Civita connections associated wi th a metric deformation $t \mapsto g(t)$ of $g$. In this case, if $h:=g^{\prime}(0)$ is the 
$1-j$ et of the metric deformation of $g$, then

$$
\begin{aligned}
2 R^{\prime}(x, y) z= & (D D h)(x, y ; z,-)^{\#}+(D D h)(x, z ; y,-)^{\#}-(D D h)(x,-; y, z)^{\#} \\
& -(D D h)(y, x ; z,-)^{\#}-(D D h)(y, z ; x,-)^{\#}+(D D h)(y,-; x, z)^{\#} .
\end{aligned}
$$

Define $\square: S^{2}(M) \rightarrow S^{2} \otimes A^{1}(M)$ by

$$
2(\square h)(x, y, z):=\left(D_{x} h\right)(y, z)+\left(D_{y} h\right)(x, z)-\left(D_{z} h\right)(x, y)
$$

(compare $[2, \$ 6 . b])$. If the metric deformation $t \mapsto g(t)$ of $g$ with 1-jet $h=$ $g^{\prime}(0)$ induces the deformation $t \mapsto D^{t}$ of the Levi-Civita connection $D$ of $g$, it is well known that the tensors $C^{\prime}$ and $h$ are related by

$$
\left(D_{x} h\right)(y, z)=\left\langle C^{\prime}(x, y), z\right\rangle+\left\langle C^{\prime}(x, z), y\right\rangle
$$

or equivalently by

$$
\left\langle C^{\prime}(x, y), z\right\rangle=(\square h)(x, y, z)
$$

which we will write as $(\square h)^{\#}=C^{\prime}$.

Fix a Riemannian metric $g$ for $M$ with Levi-Civita connection $D$.

Definition. A deformation $t \mapsto D^{t}$ of $D$ through torsion free connections is covered at first order by a metric deformation $t \mapsto g(t)$ iff

(i) $D$ is the Levi-Civita connection of $g(0)$, and

(ii) $(\square h)^{\#}=C^{\prime}$, where $h=g^{\prime}(0)$ and $C^{\prime}$ is the first order difference tensor of the deformation $t \mapsto D^{t}$.

We emphasize that all that is needed for equation (5) to hold is that $g(t)$ covers $D^{t}$ at first order.

Recall the formula that if $g(t)$ is a deformation of $g$ with $g^{\prime}(0)=h$, then

$$
\begin{aligned}
R^{\prime}(h)(x, y, z, w): & =\left.(d / d t) g(t)\left(R^{t}(x, y) z, w\right)\right|_{t=0} \\
& =\left\langle R^{\prime}(x, y) z, w\right\rangle+h(R(x, y) z, w)
\end{aligned}
$$

Thus if $g(t)$ covers $D^{t}$ at first order,

$$
R^{\prime}(h)(x, y, z, w)=\left\langle r\left(C^{\prime}\right)(x, y, z), w\right\rangle+h(R(x, y), z, w)
$$

where $C^{\prime}$ is the first order difference tensor of $t \mapsto D^{t}$, and thus

$$
\begin{gathered}
K^{\prime}(x, y):=\left.(d / d t)\left(K^{t}(x, y)\right)\right|_{t=0}=\left\langle r\left(C^{\prime}\right)(x, y, y), x\right\rangle+b(R(x, y) y, x) \\
-K(x, y)\left(b(x, x) b(y, y)-(b(x, y))^{2}\right),
\end{gathered}
$$

where $\{x, y\}$ are $g$-orthonormal vectors spanning some 2-plane. Here we use the notation $K^{t}$ (resp. $K$ ) for the sectional curvature function determined by the Riemannian metric $g(t)$ (resp. $g$ ). Since $K^{t}(x, y)$ depends only on the 2-plane spanned by the tangent vectors $x$ and $y$, we can interchange $x$ and $y$ in formula (5). Hence we obtain the following curvature obstruction for 
$g(t)$ to cover $D^{t}$ at first order:

(8) $\left\langle r\left(C^{\prime}\right)(x, y, y), x\right\rangle+h(R(x, y) y, x)=\left\langle r\left(C^{\prime}\right)(y, x, x), y\right\rangle+b(R(y, x) x, y)$.

2. An application of the curvature obstruction. We use the curvature obstruction (8) to study, in the case of constant sectional curvature, the deformations (2) and (3) mentioned in the introduction, whose sum is precisely the deformation of a connection associated to a conformal deformation of a Riemannian metric.

Recall that two connections for a manifold $M$ are said to be projectively equivalent if they have the same pregeodesics. Given a 1 -form $\xi$ on $M$, let $P(\xi) \in S^{2}(M)$ be the symmetric 2-tensor defined by

$$
P(\xi)(x, y):=\xi(x) y+\xi(y) x .
$$

Weyl showed that two torsion free connections $D^{1}$ and $D^{2}$ are projectively equivalent iff there exists a 1 -form $\xi \in A^{1}(M)$ such that $D^{1}-D^{2}=P(\xi)$.

If $D^{t}$ is a smooth deformation of $D$ through connections projective to $D$, which we will call a projective deformation, then $D^{t}=D+P\left(\xi^{t}\right)$ where the map $t \mapsto \xi^{t}$ is smooth in $t$. Thus a deformation of type (2) is an example of a projective deformation. Let $\xi^{\prime}:=\left.(d / d t) \xi^{t}\right|_{t=0}$ be the first order difference 1 -form of the projective deformation $D^{t}$ of $D$. Then $C^{\prime}=P\left(\xi^{\prime}\right)$ and

$$
r\left(C^{\prime}\right)(x, y, z)=2\left(d \xi^{\prime}\right)(x, y) z+\left(D_{x} \xi^{\prime}\right)(z) y-\left(D_{y} \xi^{\prime}\right)(z) x
$$

We can now apply the curvature obstruction (8) to obtain

Proposition 1. Let $(M, g)$ be a flat, compact Riemannian manifold. Then a necessary condition for a projective deformation $D^{t}$ of the Levi-Civita connection $D$ of $g$ to be covered at first order by a metric deformation is that the first order difference 1-form $\xi^{\prime}$ of the projective deformation be a Killing 1-form, i.e., $\delta^{*} \xi^{\prime}=0$.

Corollary 2. Given $(M, g)$ flat, compact, $\xi \neq 0$ any 1 -form on $M$ that is not a Killing 1-form. Then the deformation $D_{X}^{t} Y=D_{X} Y+t(\xi(X) Y+\xi(Y) X)$ cannot be covered even at first order by a metric deformation of $g$.

Proof of Proposition 1. Suppose $g(t)$ covers $D^{t}$ at first order, $g(0)=g$. For $\{x, y\} g$-orthonormal tangent vectors,

$$
\left\langle r\left(C^{\prime}\right)(x, y, y), x\right\rangle=-\left(\delta^{*} \xi^{\prime}\right)(y, y) .
$$

Thus (8) implies $\left(\delta^{*} \xi^{\prime}\right)(x, x)=\left(\delta^{*} \xi^{\prime}\right)(y, y)$. Hence $\delta^{*} \xi^{\prime}=f g$ for some smooth function $f: M \rightarrow R$. But since $M$ is compact and has zero scalar curvature, $\delta^{*} \xi^{\prime}=0$ (see [7, Chapter 3, e specially Lemma 2.3, p. 52]). Q.E.D.

In the noncompact case in the context of Proposition 1, we see (again using [7, Chapter 3]) that the first order difference tensor $\xi^{\prime}$ must be an in- 
finitesimal conformal transformation, that is, $\delta^{*} \xi^{\prime}=f g$, where, in addition, $\Delta f=0, \Delta=d \delta+\delta d$, if the projective deformation is covered at first order by a metric deformation.

Proposition 3. Let $(M, g)$ be a complete Riemannian manifold with constant sectional curvature $c \neq 0$. Let $\xi$ be a nontrivial Killing 1-form, i.e., $\delta^{*} \xi=0$. Then $D^{t}=D+t P(\xi)$ is a deformation of type (2) which cannot be covered even at first order by a metric deformation of $g$.

Proof. Suppose $g(t)$ is a metric deformation of $g$ covering $D^{t}$ at first order. For $\{x, y\} g$-orthonormal, since $\delta^{*} \xi=0$ we have

$$
\left\langle r\left(C^{\prime}\right)(x, y, y), x\right\rangle+h(R(x, y) y, x)=-\delta^{*} \xi(y, y)+c h(x, x)=c h(x, x) .
$$

Thus (8) implies $h(x, x)=h(y, y)$. Hence $h=f g$ where $f: M \rightarrow R$ is smooth. But then equation (5) in this particular case implies

$$
x(f)\langle y, z\rangle=2 \xi(x)\langle y, z\rangle+\xi(y)\langle x, z\rangle+\xi(z)\langle x, y\rangle
$$

for all $x, y$, and $z$. Taking $x=y=z \neq 0, x(f)=4 \xi(x)$ which implies $d f=4 \xi$. Taking unit vectors $x, y=z$ with $\langle x, y\rangle=0$, we have $x(f)=2 \xi(x)$ which implies $d f=2 \xi$. Hence $d f=0$, so $f$ is constant. But then $D h=0$, since $h=$ $f g$, so $C^{\prime}=(\square h)^{\#}=0$, a contradiction. Thus $D^{t}$ cannot be covered even at first order by a metric deformation. Q.E.D.

We now study deformations of type (3), $D_{X}^{t} Y=D_{X} Y+\operatorname{tg}(X, Y) Z$. Let $\eta$ be the 1-form associated to $Z$ be the metric $g$, i.e., $\eta(x)=g(Z(\pi(x)), x)$. Recall that if $(M, g)$ satisfies the condition $K \geq 0$ (or $K \leq 0$ ), then for $\{x, y\}$ $g$-orthonormal, $K(x, y)=0$ implies $R(x, y) y=R(y, x) x=0$. If a metric deformation $g(t)$ of $g$ covers the above type (3) deformation at first order, then $\left\langle r\left(C^{\prime}\right)(x, y, y), x\right\rangle=\left\langle D_{x} Z, x\right\rangle$. Thus if $K \geq 0$ and $K(P)=0$, for a g-orthonormal basis $\{x, y\}$ for $P$, the curvature obstruction (8) implies

$$
\left\langle D_{x} Z, x\right\rangle=\left\langle D_{y} Z, y\right\rangle
$$

or equivalently, $\delta^{*} \eta(x, x)=\delta^{*} \eta(y, y)$. Applying observation (9) to the basis $\{(x+y) / \sqrt{2},(x-y) / \sqrt{2}\}$, a calculation shows

$$
\delta^{*} \eta(x, y)=0
$$

Thus in the case $K \geq 0$ (or $K \leq 0$ ), the possible choices of $Z$ so that the deformation (3) of connections can be covered by a metric deformation are limited by equations (9) and (10). In particular, we have the same conclusion as in Proposition 1 in

Proposition 4. Suppose $(M, g)$ is flat, compact. If the deformation $D_{X}^{t} Y$ $=D_{X} Y+\operatorname{tg}(X, Y) Z$ can be covered at first order by a metric deformation, then $Z$ is a Killing vector field. 
Proof. Equations (9) and (10) imply $\delta^{*} \eta=f g$ for some smooth $f: M \rightarrow R$ ( $\eta$ as above). Then by the proof of Proposition $1, Z=\eta^{\#}$ is a Killing vector field. Q.E.D.

Like Proposition 3, we have

Proposition 5. Suppose $(M, g)$ is complete with constant sectional curvature $c \neq 0$. Let $Z \neq 0$ be a Killing vector field. Then the deformation of connections, $D_{X}^{t} Y=D_{X} Y+\operatorname{tg}(X, Y) Z$, cannot be covered even at first order by a metric deformation of $g$.

Proof. Since $Z$ is Killing, $\left\langle D_{x} Z, x\right\rangle=0$ for all $x \in T M$. Hence equation (8) applied to any two $g$-orthonormal vectors $x$ and $y$ reduces to $\operatorname{ch}(x, x)=$ $c h(y, y)$. Hence $h=f g$ for some smooth $f: M \rightarrow R$. Then by equation (5) and the equality $h=f g$, for all $x, y, w \in M_{p}$, and $p \in M$,

$$
\left(D_{x} h\right)(y, w)=x(f)\langle y, w\rangle=\langle x, y\rangle\langle Z(p), w\rangle+\langle x, w\rangle\langle Z(p), y\rangle .
$$

If $Z(p)=0$, evidently $\left.D h\right|_{p}=d f(p)=0$. Thus suppose $Z(p) \neq 0$. Given any $0 \neq x \in M_{p}$, choose $y=w$ to be a unit vector with $\langle x, y\rangle=0$. By (11) for this choice of $x$ and $y=w, x(f)=0$. Thus $d f(p)=0$. We have thus shown that $d f=0$ on $M$. Then $D h=0$ because $h=f g$, and hence $C^{\prime}=(\square h)^{\#}=0$. But this implies $Z$ vanishes identically, a contradiction. Q.E.D.

\section{REFERENCES}

1. T. Aubin, Métriques riemannienes et courbure, J. Differential Geometry 4 (1970), 383-424. MR 43 \#5452.

2. M. Berger and D. Ebin, Some decompositions of the space of symmetric tensors on a Riemannian manifold, J. Differential Geometry 3 (1969), 379-392. MR 42 \#993.

3. J.-P. Bourguignon, Transformations infinitésimales conformes fermées des variétés riemannienes connexes complètes, C. R. Acad. Sci. Paris Sér. A-B 270 (1970), A1593-A1596. MR 42 \#5192.

4. P. E. Ehrlich, Local convex deformations of Ricci and sectional curvature on compact Riemannian manifolds, Proc. Sympos. Pure Math., vol. 27, Part I, Amer. Math. Soc., Providence, R. I., 1975, 69-72.

5. N. J. Hicks, Notes on differential geometry, Van Nostrand Math. Studies, no. 3, Van Nostrand, Princeton, N. J., 1965. MR 31 \#3936.

6. M. Obata, The conjectures on conformal transformations of Riemannian manifolds, J. Differential Geometry 6 (1971/72), 247-258. MR 46 \#2601.

7. K. Yano, Integral formulas in Riemannian geometry, Pure and Appl. Math., no. 1, Dekker, New York, 1970. MR $44 \# 2174$.

DEPARTMENT OF MATHEMATICS, SUNY, STONY BROOK, NEW YORK 11790

Current address: Mathematisches Institut der Universität Bonn, 53 Bonn, Wegelerstrasse 10 , West Germany 